\title{
Improved dynamic cutting force model with complex coefficients at orthogonal turning
}

\author{
Jiri Drobilek ${ }^{1} \cdot$ Milos Polacek $^{1} \cdot$ Pavel Bach $^{1} \cdot$ Miroslav Janota $^{1}$ \\ Received: 27 August 2018 / Accepted: 9 April 2019 / Published online: 26 April 2019 \\ (C) Springer-Verlag London Ltd., part of Springer Nature 2019
}

\begin{abstract}
Self-excited vibration (chatter) is determined by the relation between the vibrating system and the cutting process. In current theories, the dynamic cutting force model in a form suggested by Tlusty and Polacek in the 1950s is still used. However, this model oversimplifies the dynamic cutting process by trying to express all processes using a single cutting force coefficient. Measurement results presented in this article clearly show that such simplification of the cutting process is unacceptable. This work follows upon the original measurement method by Tlusty and Polacek using controlled tool vibration. The method was intended to research the cutting process dynamics. However, the original Tlusty and Polacek method ignored the impact of the length of the workpiece surface wave. The original method is innovatively developed by taking into account the impact of the chatter frequency. The new method allows to better understand the processes occurring during dynamic cutting. As opposed to the original method, current advanced measurement equipment allows for more precise examination of the cutting process in dependence on the chatter frequency. The article shows that results obtained by the new method can be utilized for modeling the cutting force with greater precision in order to better predict the cutting process stability.
\end{abstract}

Keywords Chatter $\cdot$ Dynamic cutting force $\cdot$ Complex cutting coefficient $\cdot$ Stability limit prediction $\cdot$ Orthogonal turning

\section{Introduction}

Depending on the parameters of the mechanical structures of the machine and of the cutting process, turning can either be chatter-free or the tool can start vibrating during cutting even without any external excitation force. This process is known as self-excited vibration or chatter. The boundary between the two conditions is called the stability limit. The stability limit can be visualized as a borderline dividing the cutting

Jiri Drobilek

drobilek.jiri@sub-tos.cz

Milos Polacek

m.polacek@rcmt.cvut.cz

Pavel Bach

p.bach@rcmt.cvut.cz

Miroslav Janota

m.janota@ remt.cvut.cz

1 CTU, Faculty of Mechanical Engineering, Research Center of Manufacturing Technology (RCMT), Horska 3, Prague 2128 00, Czech Republic conditions in two groups. One group includes conditions under which machining will always be chatter-free, while the other group includes conditions for unstable cutting. The chip width $b$ is considered to be a significant parameter defining the stability limit in practical terms. In practice, it is often represented in dependence on selected parameters, such as cutting speed, chatter frequency, or other technological parameters relevant for the particular case (workpiece material, tool parameters, etc.). Such representation is known as the stability lobe diagram.

Unstable cutting conditions may induce massive chatter which poses a risk of not only bad quality of the machined surface but also tool or even machine damage. Self-excited vibration can arise in dependence on the resilience of the machine-tool-workpiece system, on the dependence of the resulting interaction force between the tool and the workpiece on the system chatter, and on technological parameters of turning. By this, we mean the impact of the force(s) between the tool and the workpiece on the magnitude, direction, and phase of the excited chatter, considering also the retroaction between such force and the system chatter.

Research into suppressing self-excited vibration dates back to the $1950 \mathrm{~s}$. With regard to the level of computational and experimental technology available at that 
time, research of self-excited vibration had to be simplified significantly; focus was given to improve parameters of mechanical vibrating systems and to optimize the parameters of the cutting process.

As a general rule, stability of the cutting process depends on the mechanical structure dynamics and on the dynamic cutting process. A dynamic process in this context means a process variable in time.

One aspect cannot be prioritized over the other when defining the conditions of chatter-free machining.

The mechanical structure of the machine consists of different elements. Some can be represented as a mass, other as a spring or a damper. The dynamic behavior of the system is described using transfer functions. In practice, the frequency transfer function is the most widely used, describing chatter induced by the force interacting between the tool and the workpiece. The direction of the chatter and the direction of the acting force are considered.

However, dynamic behavior of a complex mechanical structure cannot be described merely on the basis of measured transfer functions in the directions of the coordinate system axes, which is often the general practice. The directions of individual modal shapes also need to be taken into account. These directions and the direction of the acting force determine the directional factor. The directional factor indicates the contribution of different modal shapes to the overall displacement in a specific direction, see Fig. 1. If a dynamic cutting force is applied in a certain direction, then, its orthogonal projections into the directions of each modal shape will cause a displacement in that direction. Depending on the dynamic properties of the different shapes, the displacement will be of a certain size. The sum of these displacement projections in the direction of the normal $Y$ will be decisive for the occurrence of an unstable cutting process.

For a more detailed explanation of the above, see [1] or [2]. These publications provide a very clear overview of the current status of chatter research.

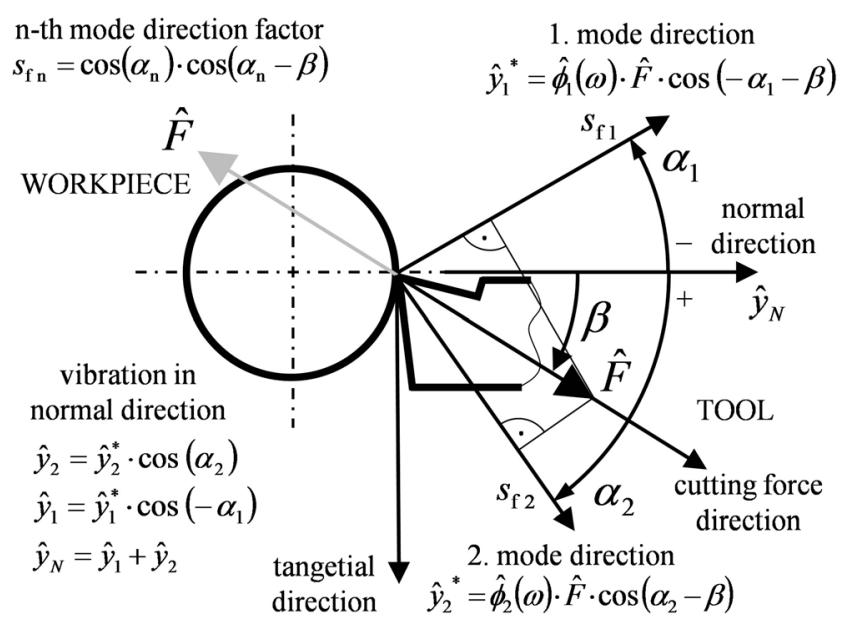

Fig. 1 Directional orientation
The dynamic properties of a mechanical structure are determined by its stiffness, natural frequency, and damping of the different modal shapes. Directional factors, together with other modal shape parameters, express the contribution of each modal shape to the occurrence of chatter.

The directional factor influences stability in all types of machining. The impact of this particular parameter can be seen, e.g., in milling or drilling, the reason being that these technologies involve a rotating dynamic cutting force. If the decisive factor of stability is a rigid vibrating system (i.e., the machine-supporting structure), the stability of the cutting process may increase or decrease depending on the angle between the cutting force and the different modal shapes.

At a low cutting speed and a specific chip width $b$, the rotating tool will smoothly pass between stable and unstable cutting and the waviness will appear only in particular areas of the workpiece surface.

This article will further presume that today's methods of identifying the dynamic behavior of machine structures are very advanced and that there can be no doubts about their reliability and accuracy for the purposes of determining the stability limit.

The content of this article focuses on the cutting process, namely, on the cutting process dynamic force arising between the tool and the workpiece due to vibrations.

Until the 1950s, research of self-excited vibration concentrated on identifying its causes in terms of technology. In the 1950s, the professional public recognized two principles explaining and describing cutting process instability. The principles are position feedback and regeneration [3]. Position feedback was found to be the cause of self-excited vibration. Position feedback showed that the vibrating system has its role as much as the acting cutting force. Further in this article, we will concentrate solely on the regenerative principle.

According to the regenerative principle, apart from the tool vibration force there is also the wave cutting force. The tool cuts waves created during the previous revolution or-in the case of milling - during the preceding cutting-edge passage.

The waves have their amplitude and wavelength determined by the chatter and cutting speed. Waves that are being cut show a phase shift by angle $\psi$ against the tool chatter due to the ratio between the frequency of the chatter and the frequency of rotations (i.e., the cutting speed), Fig. 2 A). If the phase shift $\psi$ equals zero (the ratio between the chatter and rotation frequencies is an integral number, Fig. 2 B), no unstable cut may occur according to the regenerative principle.

A general principle applies for self-excited vibration that the mechanical structure and the dynamic cutting process form a closed system. For an unstable cut to occur, the system must be able to set a non-zero phase shift between the tool vibration and the resulting dynamic cutting force. For regenerative chatter, it is the phase shift $\psi$ 


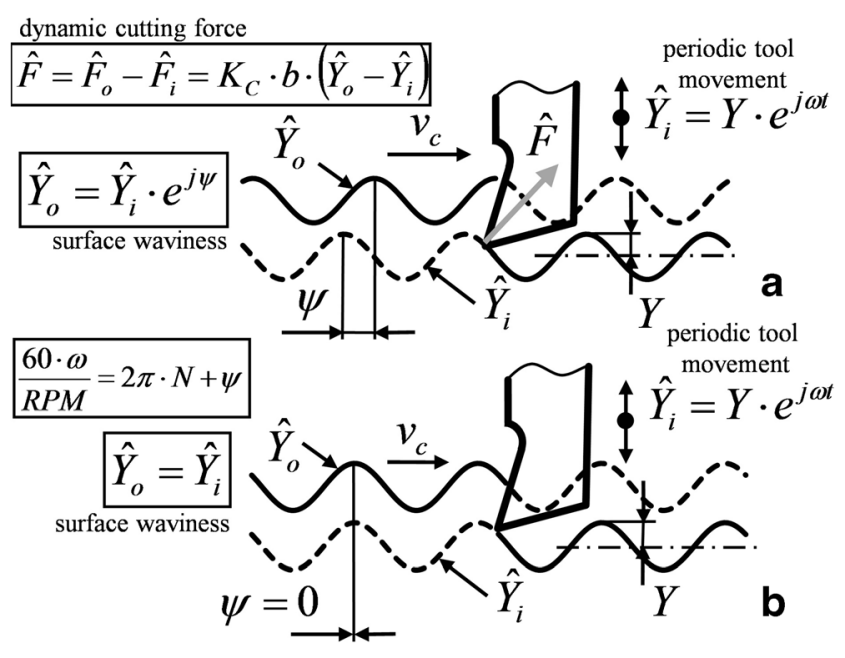

Fig. 2 Regenerative dynamic cutting force

which is set by changing the ratio between the frequencies of the chatter and of the turning rotations.

The phase shift is associated with a certain frequency of tool vibration and of turning rotations. There is a different phase shift corresponding to each frequency, but for the given system, such frequency is set that gives rise to chatter with the minimum chip width $b$. It is mainly the regenerative principle that is used to define the stability limit because the established dynamic force model extremely simplifies the description of the dynamic cutting process. It allows to determine the influence of the vibrating system on the stability limit even for systems with a single modal shape. The regenerative principle started to be used in practice in machine tool design in order to increase their resistance to chatter.

In the late 1960s, a cooperative research of several international research labs took place under the umbrella of CIRP, whose aim was to describe with more precision the equations then used for defining the stability limit and to establish a methodology for measuring the dynamic cutting force analogically to measure the dynamic behavior of a mechanical structure. Using specific measurement and evaluation methods, dynamic cutting force coefficients were established for tangential and normal directions, Fig. 1. These were complex coefficients, i.e., they contained information about the amplitude ratio and about the phase shift between the relevant force component and the tool vibrations in the direction of the normal $Y$, similar to the values of the complex transfer function of a mechanical structure.

In spite of the effort to maintain uniform measurement conditions across all the labs, the results from individual laboratories showed considerable dispersion of values [4].

Numerous other authors also attempted research in the field ([5-10]), but due to demanding requirements on hardware and time needed for the experiment, they sought a practicable way of simplifying and accelerating the acquisition of data. However, the simplifications only resulted in larger or lesser inexplicable deviations from the measurement results obtained by the cooperative research. The majority of authors concentrated mainly on identifying the complex coefficients without investigating their further use for calculation of the stability limit.

The term "complex" may appear somewhat unclear, but it can be easily explained.

Let us admit that the dynamic force $F$ is not only directly proportional to chatter amplitude $Y$, Fig. $3 \mathrm{~A}$ ), but that there is also a time shift between the amplitude and the force, Fig. 3 B). In reality, the dynamic cutting force is directly proportional to the cross-section of the cut layer. For the sake of simplicity, let us presume that the cut width $b$ is constant and therefore, the dynamic force depends only on the amplitude $Y$. If therefore the movement of the tool $Y$ varies periodically in time, the force $F$ will also vary with the same period, but the peak of each value will occur at different moments due to time delay. The existing time delay can be expressed with the use of chatter frequency as the phase of the force $F$ against the amplitude $Y$. Using a complex number, we can thus express both the magnitude of force and the considered phase at the same time. The magnitude of the force (its amplitude) is defined by the cutting resistance, while the phase is determined, e.g., by the shift between the waves and the tool chatter or by time difference due to the properties of the material whose structure goes through different states during machining.

The expression "complex" can thus be understood as encompassing both amplitude and phase-shifted. However, the considered time delay can be analyzed also directly in the domain of time. Semi-discretization methods can be applied when looking for a solution [11]. In principle, these methods consider time delays with various dependencies. Complex dynamic coefficients are marginally discussed also in one of the sub-chapters [2].

The current dynamic cutting force model is based on two basic dynamic forces: the force from the tool vibration (inner

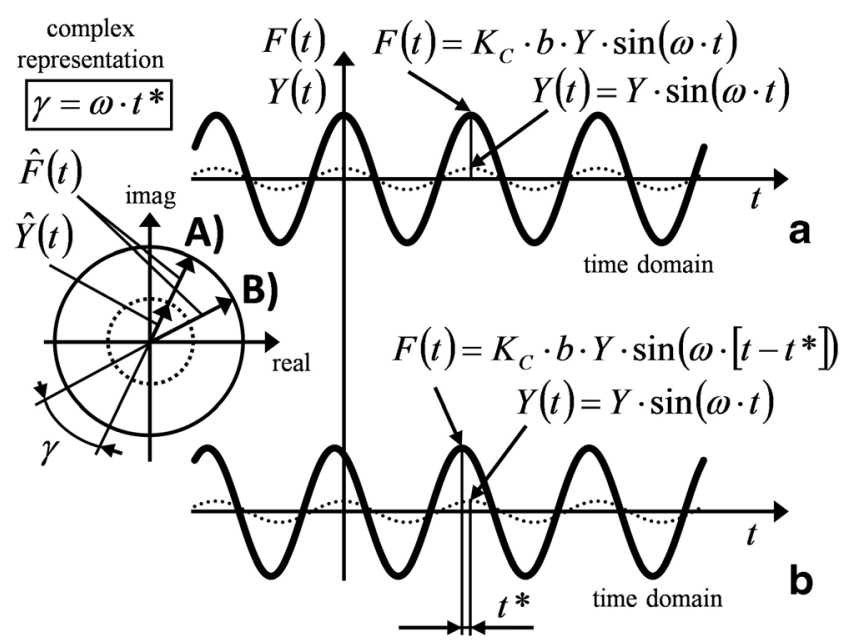

Fig. 3 Complex cutting force 
modulation $F_{i}$ ) and the force from the wave cutting (outer modulation $F_{o}$ ). There is a phase shift between these forces corresponding to the ratio between the frequency of chatter and the frequency of rotations (phase $\psi$ ). For the sake of simplification, both these forces are often expressed as a single expression. This however obscures their original meaning.

The theory further contemplates that there is also process damping in the cutting process, which causes the stability limit to increase with decreasing cutting speed. The notion of "process damping" includes the damping force generated by the cutting process, which thus prevents the tool from chatter. The physical principle of the emergence of this force is based on the idea of a contact between the tool edge and the undulated surface of the workpiece [12]. This theory has been further expanded [13] with a force occurring due to the forcing of the rounded tool edge into the wave foot. When the radius on the cutting edge is larger than the curving at the wave foot, the material will hinder the tool movement due to lack of space, which limits the tool's maximum depth of cut. Process damping is also influenced by the tool's wear. With growing wear, the cutting edge becomes flatter, significantly reducing the tool's capacity to cut into the material and increasing the stability limit. Latest research focuses mainly on identifying the process damping forces. An elegant and simple method of identifying process damping is presented in [14]. This method requires no expensive measuring device to measure damping. Damping is measured based on the maximum and minimum depths of cut.

Papers $[13,15,16]$, can also be mentioned, identify process damping using different methods.

Article [13] describes identification of the process damping force using controlled tool vibrations. During cutting, the turning tool was made to vibrate at a constant frequency with the use of a piezoelectric actuator. The frequency of the chatter against the workpiece rotation was chosen in such a way so as to ensure that a zero wave phase $\psi$ is achieved against the chatter. Therefore, only the process damping force should be acting in the cut. In reality though, both the force from the tool vibration and the force from the wave cutting persist. The two forces should mutually offset each other, since they are identical and in phase opposition. However, it cannot be confirmed with certainty that this is the case. Unless the forces are identical, their residual difference will influence the magnitude of the measured process damping.

The process damping in [13] is characterized by the process damping coefficient $C_{\mathrm{pd}}(\mathrm{N} / \mathrm{m})$ which was determined for the measured wavelength range at a constant cutting speed. However, the paper does not consider a possibility that also the other dynamic cutting forces might be dependent on chatter frequency. This presumption could be very easily verified using the designed experiment.

In [15], self-excited vibration was used to identify process damping. chatter was induced on a workpiece, clamped in overhang, of a specific shape during turning. The dynamic cutting force and workpiece chatter were measured under these conditions. This case provides the most faithful simulation of the conditions under which self-excited vibration occurs. The measured data were plotted in the complex plane and interpolated with a circle. The process damping coefficient was determined from the coordinates of the center of the circle. The evaluation method is very similar to the VUOSO method (Research Institute for Machine Tools and Machining in Prague) [4]. However, the biggest challenge to the application of this method is the chatter itself. First of all, it is necessary to find conditions under which chatter occurs. In addition, it is necessary to make sure that the tool does not jump out of the cut. Even when self-excited vibration arises, it must not only be observed repeatedly under the same conditions but it also needs to be maintained for a length of time required to make the measurements. The range of the tested frequencies is very limited, and it is determined by the dynamics of the tested workpiece. For a different range of frequencies, a new workpiece must be used and the entire process of finding the unstable cutting conditions has to be repeated again. In [15], the assumption is made that the frequency of self-excited vibration changes continuously with increasing stiffness of the vibrating system. Changing the frequency of self-excited vibration at a constant cutting speed changes the phase between the force and the vibration amplitude. This phase change is necessary for plotting the data on the complex plane. However, it is important to bear in mind that a regenerative effect cannot be achieved through a continuous change in frequency, as considered in the theory of self-excited vibrations. It is also necessary to draw attention to the results mentioned in [15]. These results clearly show that the force generated by tool vibrations and the force generated by cutting of the waves are not the same. If that were the case, the presented measured circles (and their interpolations) would be located very close to the imaginary axis or they would touch this axis. It is evident from the presented figures that the forces are not identical.

Article [16] describes the identification of process damping by means of a pulse from a modal hammer, similar to the previously used method [8]. The technological conditions of machining were chosen just below the stability limit, i.e., as close as possible to the real conditions under which chatter occurs. The excitation pulse from an impact hammer induces dynamic forces in the cut associated with tool vibrations. The disadvantage of this method is that the measured data can only be obtained during a very short time interval. The measurement time is determined by the time for which the vibration lasts. If the measured force signal is evaluated within one revolution only, the measured dynamic cutting force does not contain the wave cutting force because there was not enough time for the cutting tool to cut the waves from the surface of the workpiece during the subsequent revolution. 
Under these conditions, it is possible to identify the directly acting process damping force. However, the short measurement times and repeatability of the excitation pulses significantly affect the accuracy of the obtained results.

In some cases, a significant reduction in the stability limit may also occur within a certain cutting speed range [17].

Some authors assume $[13,18]$ that this drop is caused by the build-up of a machined material on the tip of the cutting tool. Under certain cutting conditions, the cut material adheres to the cutting edge of the tool and modifies its geometry. The new geometry creates more cutting resistance, resulting in a decrease in the stability limit. This phenomenon has not yet been taken into account in determining the stability limit.

However, the research of self-excited vibration also includes approaches and methods by which chatter can be suppressed. Therefore, these methods do not attempt to determine the cause of the problem, but they can be used to effectively suppress its effects. For an overview of the methods using this approach, see e.g., [19].

The currently used calculation of stable cutting conditions assumes that only one dynamic cutting force acts in the cut. As it turns out, when refining the accuracy of the calculation of the stability limit for real cases, it is necessary to express with greater precision not only the vibrating system but also the acting cutting forces. These forces need to be expressed depending on technology conditions. Practical experience shows that the machine's resistance to chatter is affected by the workpiece material, by the shape of the tool, and by the cutting conditions, none of which have been taken into account in the calculations until now. Many different experiments have been made, especially in the field of technology, where the occurrence of self-excited vibrations was assessed depending on various technological parameters. However, if dynamic behavior of the mechanical structure is not taken into account at the same time, it is impossible to express the influence of these individual parameters in the mathematical formulation of the stability limit calculation. The current technological knowledge is to a certain extent dependent on the vibrating system of the machine on which the tests were performed.

In view of the above, it should be recognized that a more accurate calculation of the stability limit can be achieved by introducing one or more additional forces (see e.g., process damping). If there are several forces acting in the cut, this can be evidenced by a measurable phase shift of the force components in two orthogonal directions. Each of these directions will include projections of the partial forces, and if these forces have different directions and are phase-shifted relative to each other, then the sums of these projections will show different phase shifts. However, phase delays cannot be observed under static conditions. It is necessary that the forces be measured under conditions under which they occur, i.e., during tool vibration.

\section{Stability limit computation}

The cutting process stability limit can be called a "steady state" when the amplitude of the undesirable vibrations of the tool remains constant. Since the movement of the tool is periodic over time, it can be expressed using complex numbers instead of goniometric functions.

In this case, complex numbers can make numerical operations with the considered phase shifts considerably easier. Unless indicated otherwise, the " $\wedge$ " symbol will be used in the text below to denote a complex number.

$\hat{A}(t)=A \cdot e^{j \beta} \cdot e^{j \omega \cdot t}=\operatorname{re}(\hat{A})+j \operatorname{im}(\hat{A})$

The generally accepted formula expressing the dynamic component of the cutting force according to the regenerative principle is as follows:

$$
\hat{F}=K_{\mathrm{C}} \cdot b \cdot\left(\hat{Y}_{o}^{\hat{\prime}}-\hat{Y}\right)
$$

where

$$
\begin{aligned}
& \hat{F} \quad \text { is the dynamic cutting force }(\mathrm{N}) \text {; } \\
& \hat{Y}_{0}=\hat{Y} \cdot e^{-j \psi} \text { is the workpiece surface waviness in the } \\
& \text { normal direction (m); } \\
& \hat{Y}=Y \cdot e^{j \omega t} \quad \text { is the tool vibration in the normal direction } \\
& \text { (m); } \\
& K_{\mathrm{C}} \quad \text { is the static cutting resistance in the normal } \\
& \text { direction }\left(\mathrm{N} / \mathrm{m}^{2}\right) \text {; } \\
& b \quad \text { is the width of the removed layer (cut width) } \\
& \begin{array}{ll}
\text { and } \omega & (\mathrm{m}) ; \\
\text { is the angular frequency of tool vibrations }
\end{array} \\
& (\mathrm{rad} / \mathrm{s}) \text {. }
\end{aligned}
$$

The expression $\psi$ shows the phase shift of tool vibration relative to workpiece surface waviness, and its value is determined by the rotation speed equation.

$\frac{60 \cdot \omega}{2 \pi \cdot n}=N+\frac{\psi}{2 \pi}$

where

$N \quad$ is the number of whole waves on workpiece surface (-);

and $n \quad$ is the rotation speed (rpm).

Alternatively, also, the cutting speed $v_{\mathrm{c}}(\mathrm{m} / \mathrm{min})$ can be used in the rotation speed equation.

$\frac{30 \cdot \omega \cdot D}{v_{c}}=N+\frac{\psi}{2 \pi}$

where $D$ is the workpiece diameter (m).

Thus, the dynamic cutting force model consists of two forces which are dependent only on tool vibration in the 
normal direction and on the cutting resistance $K_{\mathrm{C}}$, which is identical for both forces. The force containing the phase $\psi$ is a dynamic force generated by the cutting of the waves, and it is a force causing further vibrations.

The sign convention used in Eq. (2) is governed by the direction of the normal to the machined surface where the normal is oriented outwards from the workpiece. A positive surface waviness increases the dynamic cutting force, while a positive tool deflection decreases it.

Equations (2) and (3), or (4), can be applied to machining using a single edge tool. In the case of multi-edged tools (e.g., milling cutters), the equations can be easily modified by introducing the appropriate number of cutting edges. Eq. (2) applies provided that the cutting resistance $K_{\mathrm{C}}$ does not depend on the wavelength of the vibration. It should be noted that the phase $\psi$ is determined from Eq. (3), or (4), simply by changing the wavelength. By using the dynamic cutting force (2) and complex transfer functions of the mechanical structure $\hat{\varphi}_{o}(\omega)$, the known equation for the stability limit can be derived. The "o" in the equation indicates the orientation of the transfer function in the direction of the normal.

$b=\frac{-1}{2 \cdot K_{\mathrm{C}} \cdot \mathrm{re}\left(\hat{\varphi}_{o}\right)_{\mathrm{NEG}}}$

The stability limit in this case is determined by the cutting resistance $K_{\mathrm{C}}$ and by negative values of the real component $\hat{\varphi}_{o}$. The transfer function $\hat{\varphi}_{o}$ includes all considered modal shapes including their orientation, and it also expresses the orientation of the dynamic cutting force, see Fig. 1.

However, Eq. (2) is just the most simplified approximation of what really happens in the cut. In the early days of chatter research, only one resulting dynamic cutting force was measured and one real dynamic coefficient was determined $K$. This coefficient has been replaced in practice by static cutting resistance $K_{\mathrm{C}}$ for easier understanding. After CIRP initiated work on its research task, it was necessary to decide how to measure the dynamic coefficient properly. Prof. Peters [20] recommended that CIRP use a measurement methodology developed at VUOSO, which was based on controlled induced vibrations of the cutting tool. Using this methodology, the dynamic cutting force could be divided into two components, see Eq. (6), and each of these components examined separately.

$\hat{F}=\hat{F}_{i}+\hat{F}_{o}$

The first term of the right-hand side of the equation describes the force occurring as a result of tool vibration (referred to as inner modulation, $\hat{F}_{i}=b \cdot \hat{K}_{i} \cdot \hat{Y}_{i}$ ). The second term expresses the wave cutting force (referred to as outer modulation, $\hat{F}_{o}=b \cdot \hat{K}_{o} \cdot \hat{Y}_{o}$ ). Both force components are independent of each other and can occur separately. Equation
(6) describes a circle in a complex plane and the coefficients $\hat{K}_{i}$ and $\hat{K}_{o}$ can be determined from its geometric parameters. However, the scope and accuracy of the measurements conducted by CIRP were limited by the technical equipment available at that time. Only a limited number of data points could be obtained by measurements, and experimental identification of the entire dynamic force circle was thus impossible. The coefficients $\hat{K}_{i}$ and $\hat{K}_{o}$ were determined for both the normal and the tangential directions from the interpolation circle used to interpolate these few data points. These coefficients were complex numbers, i.e., they comprised a real and an imaginary component, and were assumed to be independent of the wavelength of the vibration.

\section{Dynamic cutting coefficient}

Equation (2) can be used to express the cutting resistance in dependence on the frequency of vibrations as (7). This is the simplest expression of the dynamic cutting force coefficient. Due to the phase $\psi$, it becomes a complex expression and it can be represented in the complex plane as a circle, see Fig. 4 A). The circle passes through the origin of the complex plane and its center lies on the real axis.

$\frac{\hat{F}}{b \cdot \hat{Y}}=K_{\mathrm{C}} \cdot\left(e^{-j \psi}-1\right)$

In the case of unstable cutting, forces on the face and the back of the tool are formed as a result of plunging of the tool into the material. However, when waves are cut, a force arises due to other reasons and it can be therefore assumed that the coefficient $K_{\mathrm{C}}$ may not be the same for both forces.

For the case of a force described by two mutually different coefficients, and let us assume for the sake of simplicity only the real coefficients $K_{i}$ and $K_{o}$ in Eq. (8), the cutting force in the complex plane will again be represented by a circle centered on the real axis, but this time, the circle will no longer pass through the origin, Fig. 4 B).

$\frac{\hat{F}}{b \cdot \hat{Y}}=K_{o} \cdot e^{-j \psi}-K_{i}$

If the two coefficients are complex numbers, see the coefficients $\hat{K}_{i}$ and $\hat{K}_{o}$ from CIRP research, the circle can lie anywhere in the complex plane and, moreover, it will be rotated due to the phase of the complex coefficient $\hat{K}_{o}$, Fig. 4 C).

Therefore, for a certain $\psi$, the relevant point of Eq. (7) lies on a helix rather than on a circle, see Fig. 5 A). The circle becomes only one of the projections of the helix to the relevant plane. In fact, by continuously changing the frequency of the vibrations, the number of waves changes continuously from 
Fig. 4 Circles of different cutting coefficients

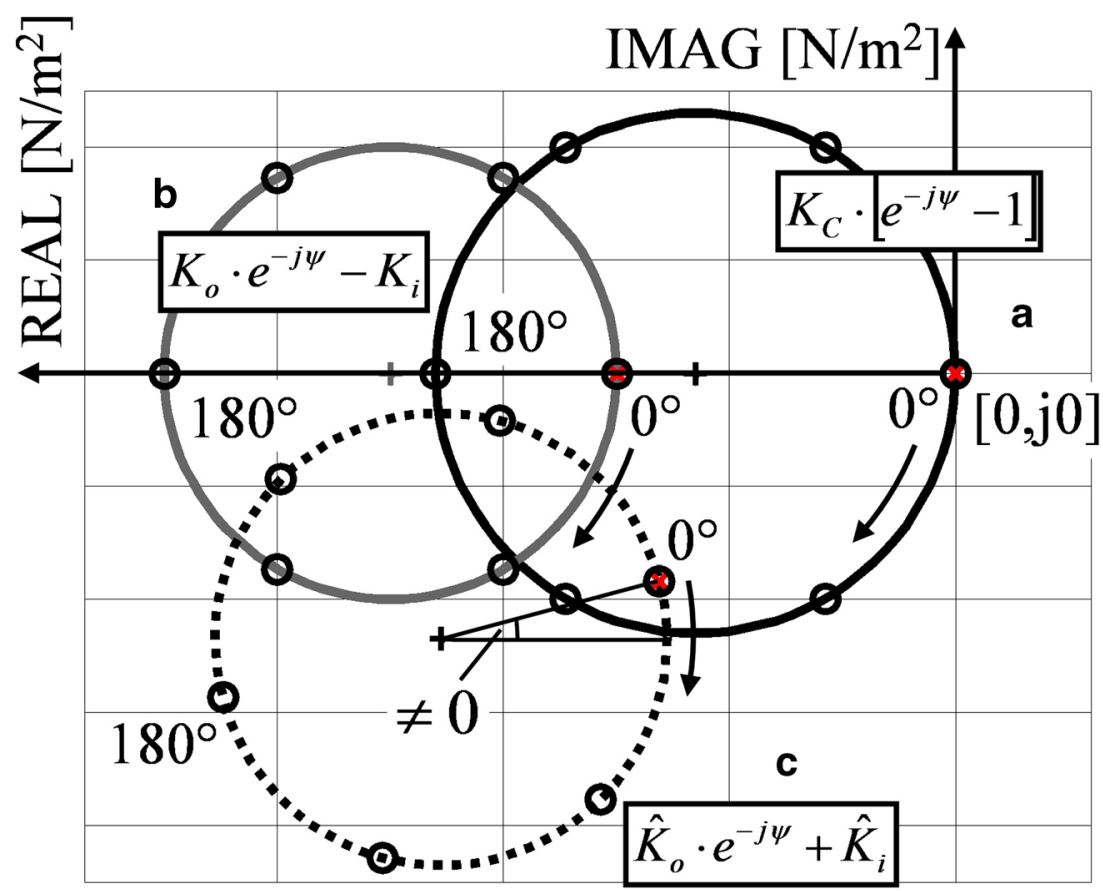

one value to another. This property is not taken into account in the theory using a circle. The other projections of the helix show the real and imaginary components as a function of vibration frequency. An example of one helix thread for a particular number of waves is shown, see Figs. 6 and 7. The centers of the circles, or rather the center lines of the individual threads, lie on a single line that passes through the coordinate $\left[-K_{\mathrm{C}}, j 0\right]$, i.e., the negative real axis. The thread starting and ending points (originally a point on a circle) for $\psi=0$ and $\psi=$ $2 \pi$ also lie on one straight line. The projection of this line is a point at the origin of the complex plane. Equation (7) should be valid at high cutting speeds where the process damping effect is negligible.

At low cutting speeds, the effect of process damping becomes stronger. It has been confirmed [12] that process
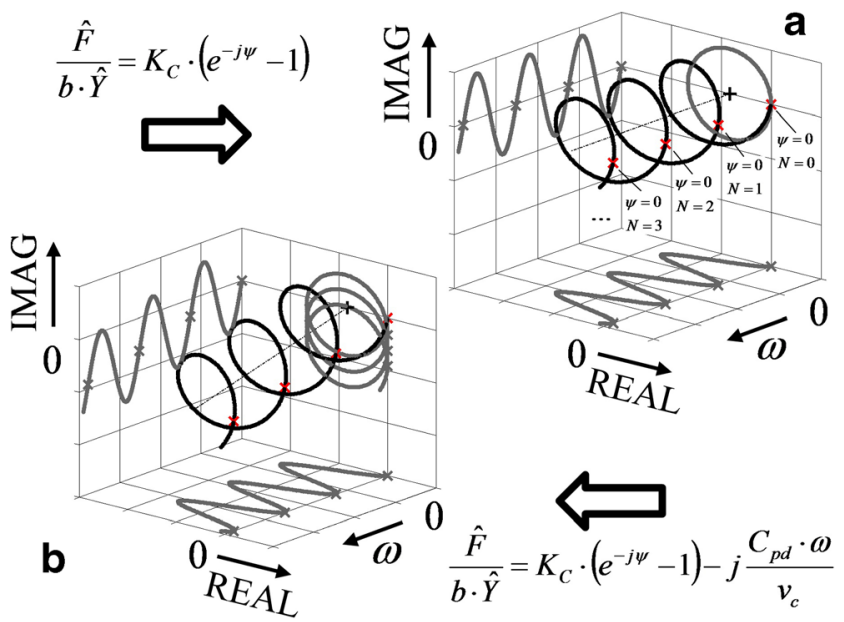

Fig. 5 Helix of dynamic cutting coefficient damping depends on the wavelength, i.e., the ratio of vibration frequency to cutting speed, or revolution speed.

This needs to be taken into account and the equation for dynamic cutting resistance needs to be extended with the contribution of process damping, e.g., to [13].

$\frac{\hat{F}}{b \cdot \hat{Y}}=K_{C} \cdot\left(e^{-j \psi}-1\right)-j \frac{C_{p d} \cdot \omega}{v_{c}}$

where

$C_{\mathrm{pd}} \quad$ is the process damping coefficient $(\mathrm{N} / \mathrm{m})$; and $v_{\mathrm{c}}$ is the cutting speed $(\mathrm{m} / \mathrm{s})$.

There exists a hypothesis that the process damping force is due to the contact between the tool flank and the undulated surface. In order for this contact to occur, the slope of the wave must be greater than clearance angle of the tool. The slope of

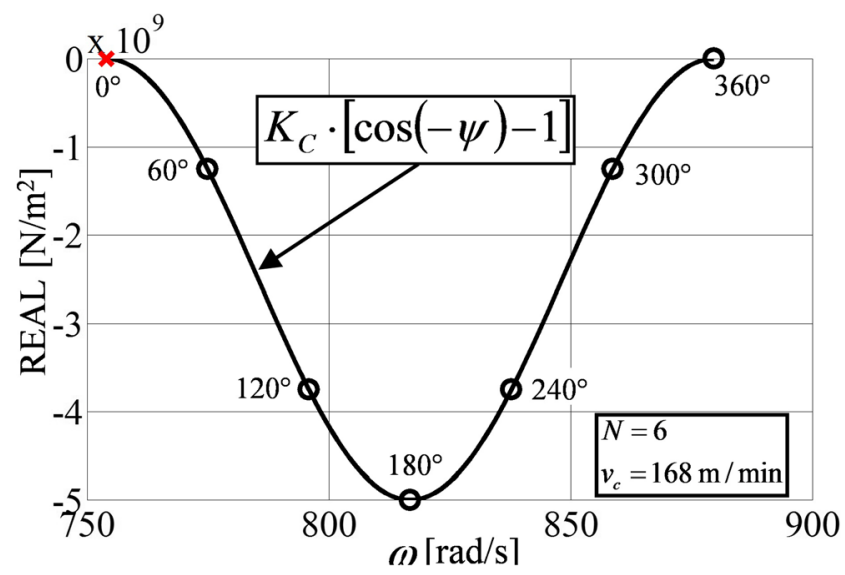

Fig. 6 Real component of standard model 


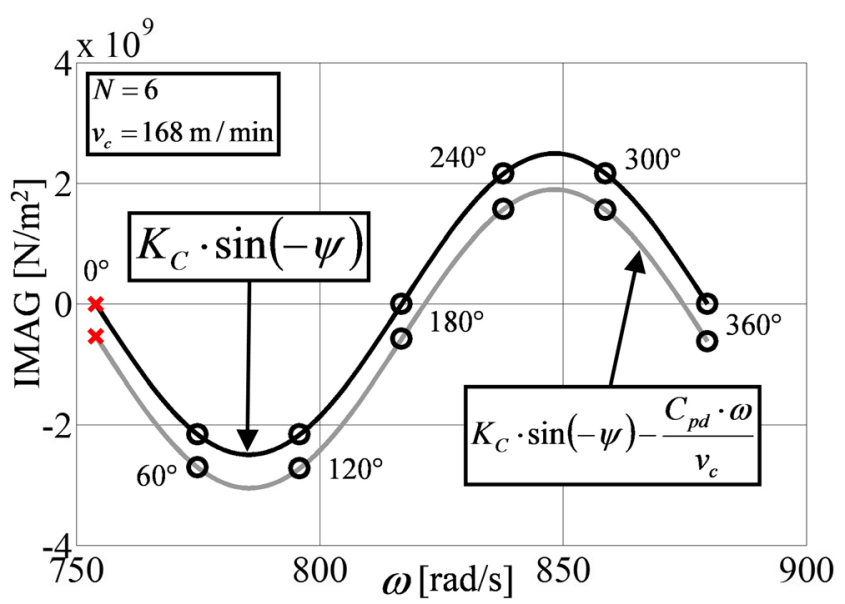

Fig. 7 Imaginary component of standard model

the wave is determined by the velocity of vibration. If the amplitude of the vibrations changes periodically, the velocity of vibration becomes phase-shifted by $90^{\circ}$ due to the derivative. Therefore, the contribution of process damping in Eq. (9) is an imaginary component.

As can be seen from Eq. (9), the contribution of process damping only affects the imaginary component of cutting resistance. Thus, the helix will sink or rise in the direction of the imaginary axis exactly according to the value of the process damping coefficient, Fig. 5 B). In this case, the respective helix projection no longer forms a circle because the helix starting and ending points for $\psi=0$ and $\psi=2 \pi$ do not have the same position. The comparison of one helix thread is shown in Fig. 8. The course of the real component coincides with that of the real component (7) and the course of the imaginary component varies according to the value of the ratio $C_{\mathrm{pd}} / v_{\mathrm{c}}$. The center line of the helix expresses directly the dependence of process damping on the vibration frequency for a particular cutting speed.

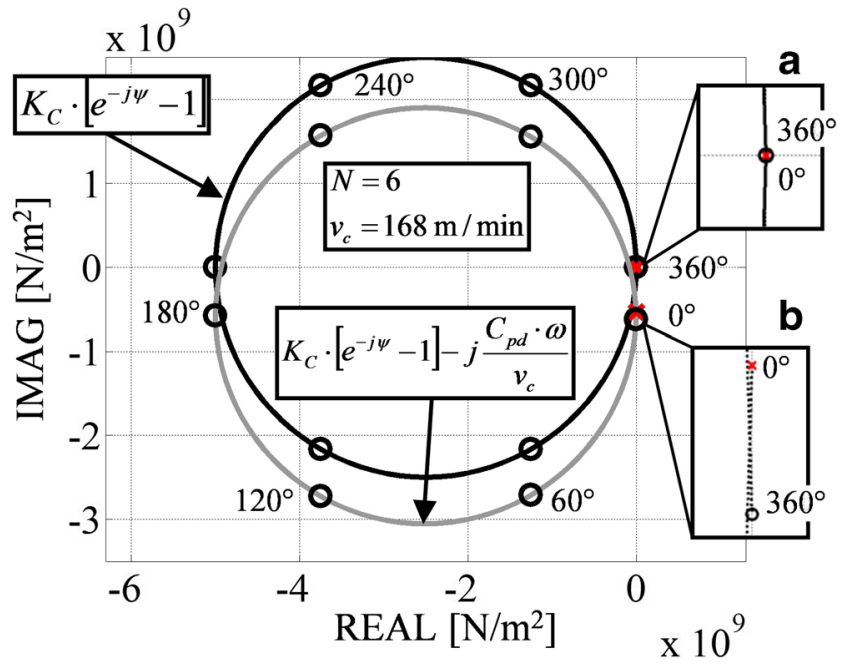

Fig. 8 Effect of process damping on standard model
In general, where the coefficients are complex and, moreover, dependent on the frequency of the vibration, the helix can have any shape, see Fig. 9.

Assuming that the existing research confirmed the existence of the above forces during dynamic cutting and these forces have been also mathematically formulated, they can be confirmed experimentally.

The aim of our research is therefore to measure the dynamic cutting forces acting in the cut similarly as was done in the 1960s, but the method used this time to identify the forces will be substantially improved. This article presents a method that makes it possible to find out what forces act in the cut. The method is based on using the identified helix to determine the dependence of the existing cutting forces on the cutting speed $v_{\mathrm{c}}(\mathrm{m} / \mathrm{s})$, but mainly also on the vibration frequency $\omega(\mathrm{rad} / \mathrm{s})$.

\section{Experiment}

To measure the dynamic cutting force, an experimental device was designed to simulate the conditions of an unstable cutting process, Fig. 10. Similarly as in the 1960s, the principle of the experiment is based on a controlled induced vibration of the tool during cutting. Quite obviously, when identification of the cutting forces is performed only on the stability limit where the system vibrates at around a single frequency, the effect of that frequency on the cutting force may not manifest at all and this solution thus yields only limited results. Process damping is thus dependent on both the frequency and the cutting speed.

The experiment was conceived as face turning on a tubular workpiece, i.e., simulation of purely orthogonal machining, Fig. 11.

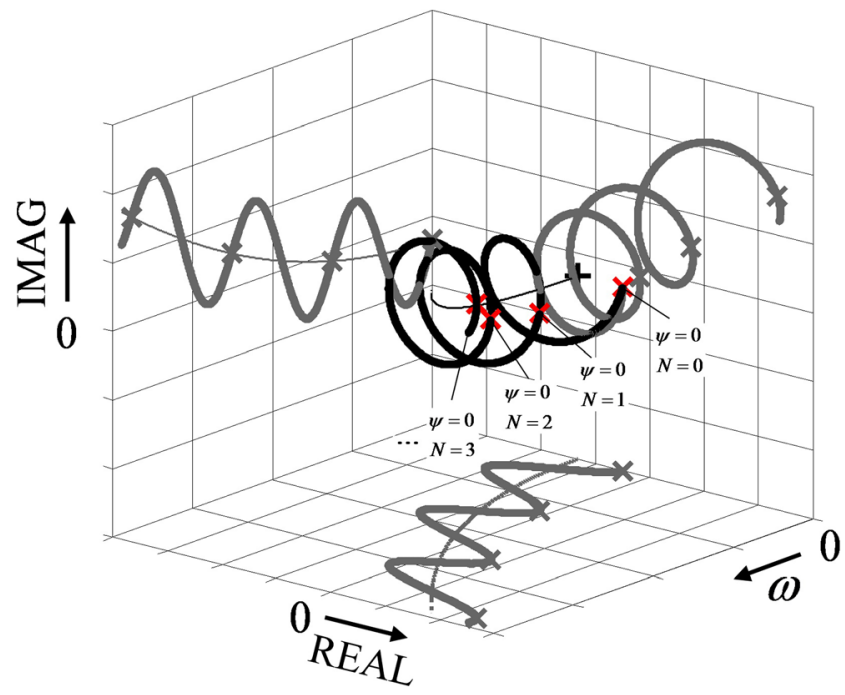

Fig. 9 Helix with complex coefficients 


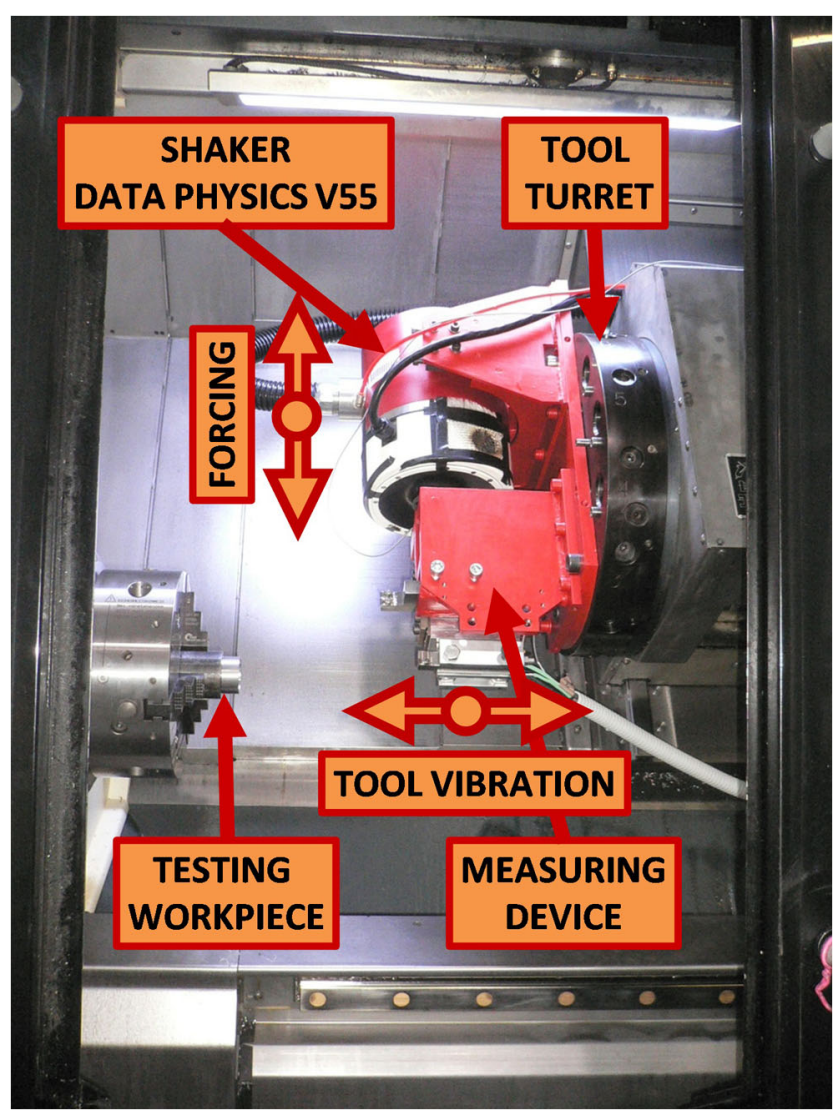

Fig. 10 Measuring device

The basis of the measuring device is a free-of-play flexible platform (parallelogram), where thin planchets act as the elastic members. The excitation is provided by the Data Physics V55 electrodynamic shaker. A test tool holder is attached to the moving part of the platform by means of three Kistler 9027C force sensors, Fig. 12.

A turning tool with a replaceable cutting insert was chosen as a test tool. This type of tool was chosen with regard to its most widespread use in chip machining technologies in both

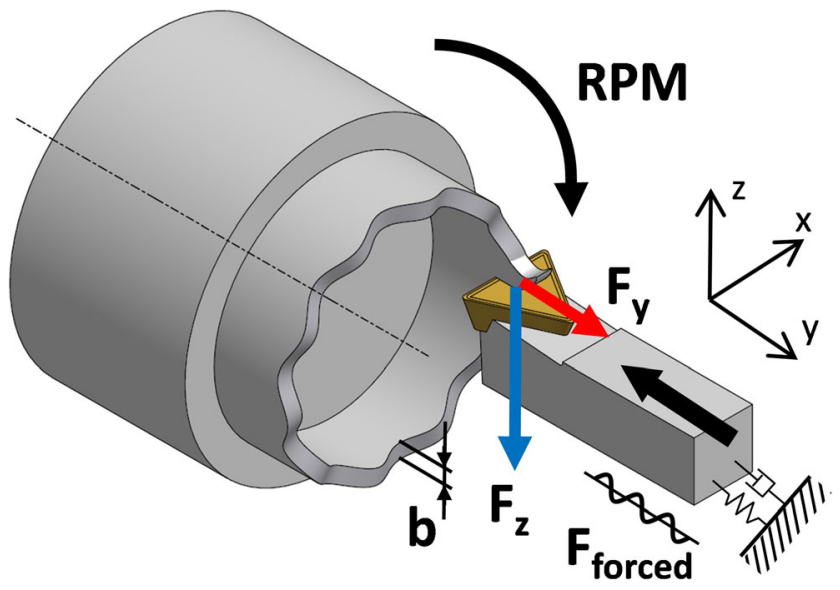

Fig. 11 Principle of test

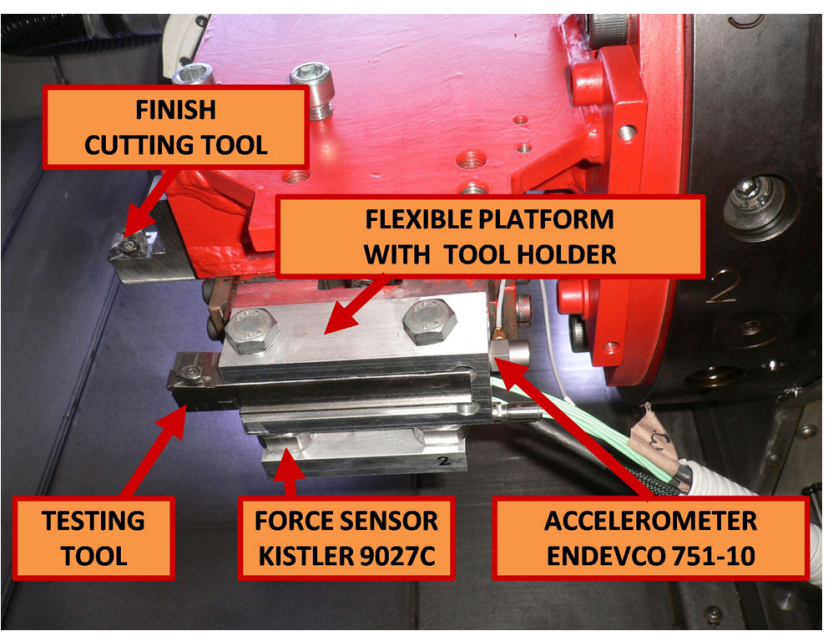

Fig. 12 Sensors on testing tool

single-piece and bulk production. The currently used cutting inserts have in most cases a modified face geometry for better chip formation. A triangular insert with a single chip breaker was selected as a test insert. The triangular shape was chosen to comply as much as possible with the orthogonal machining conditions for the selected type of workpiece (Fig. 13).

In order to eliminate a measurement error caused by the dynamic properties of the measuring device, the device was calibrated by tapping with a modal hammer (Figs. 14 and 15).

The TPMR 110304E-46, T9325, insert manufactured by PRAMET was used for the tests. The insert was clamped into a modified holder with clamping surfaces matching those of the holder type recommended by the manufacturer for the selected type of insert.

The semifinished product for the test sample was a steel rod ø $60 \mathrm{~mm}, \mathrm{CSN} 412050$, hot-drawn (CSN EN C45.0). The test samples were thin-walled tubes. The outer diameter of the tube was $45.5 \mathrm{~mm}$, the wall thickness was $1 \mathrm{~mm}$, and the length was $25 \mathrm{~mm}$. The bar stock was pre-machined to form a tube with an allowance, and the final shape of the sample
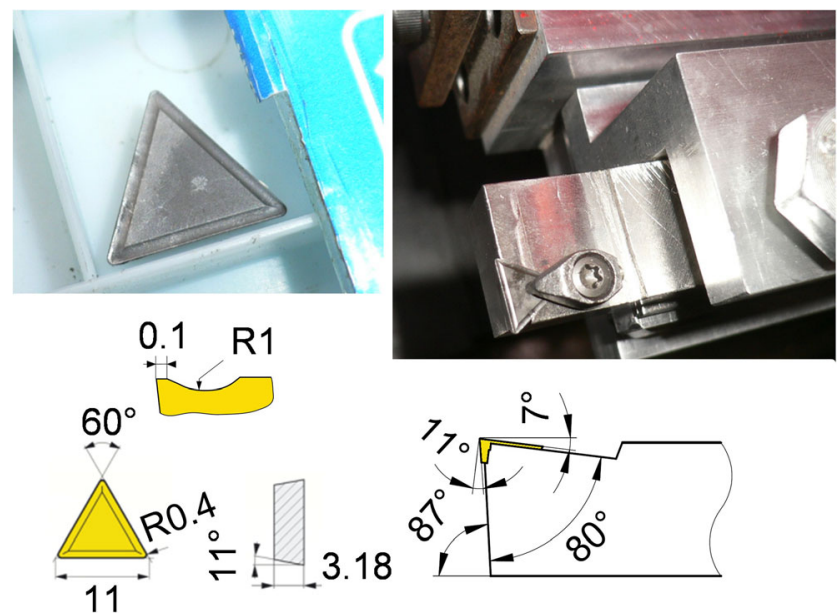

Fig. 13 Geometry of testing tool 


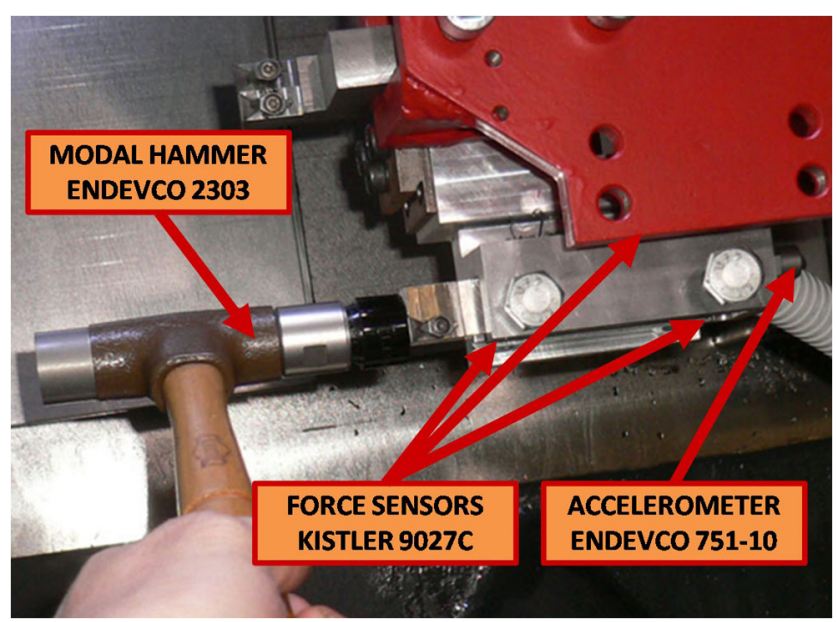

Fig. 14 Structural parameters testing

was finished on a test machine to eliminate the effect of runout of the test workpiece. Tests were performed on a DMG MORI machine, type NEF 600.

During measurement, 11 measurement channels were recorded synchronously. Each of the force sensors measured three component forces (N) $\left(F_{X 1}, F_{Y 1}, F_{Z 1}, F_{X 2}, F_{Y 2}, F_{Z 2}\right.$, $\left.F_{X 3}, F_{Y 3}, F_{Z 3}\right)$; tool holder acceleration (Acc) $\left(\mathrm{m} / \mathrm{s}^{2}\right)$ was measured using a uniaxial accelerometer, and a force sensor was attached between the shaker and the workpiece for measurement of the reference force $F_{\text {ref }}(\mathrm{N})$ applied by the shaker to the measuring device. A measuring apparatus consisting of two Brüel \& Kjaer analyzers, type Pulse 3560C, was used for data recording.

The measurement itself was carried out at a constant cutting speed. Spindle speed was not measured. It was assumed that the natural frequencies of the spindle and the drive were higher than the first resonance of the gauging fixture. The range of tested wavelengths at the face of the test sample was limited by the first natural frequency of the gauging fixture, which was about $200 \mathrm{~Hz}$.

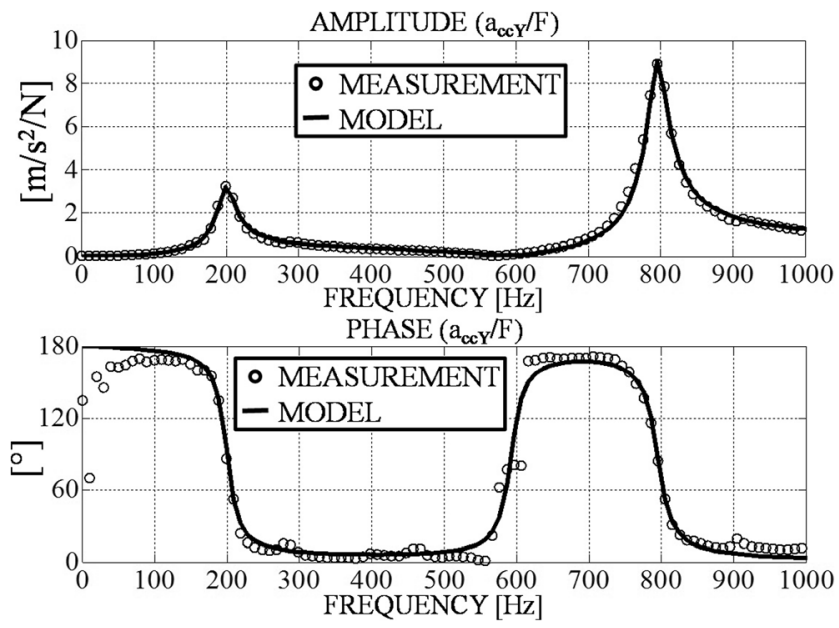

Fig. 15 Measurement device FRF in Y direction
In addition, the measured force signal contained a parasitic component of the inertial force that had to be compensated. Spectral lines corresponding to the vibration frequency were selected from the frequency spectrum of the measured signals. The ratios of the respective components and their standardization can be used to obtain points of the individual threads of a complex helix. The vibration frequency was determined for the constant cutting speed $v_{\mathrm{c}}$.

\section{Evaluation of experiment}

When refining the stability limit calculation for real cases, it is necessary to express with sufficient accuracy not only the vibrating system but also the acting cutting forces. These forces need to be expressed depending on the technological conditions and vibrations of the system. Practical experience shows that the material of the workpiece, the shape of the tool, and the cutting conditions, which were not included in the previous calculations, influence the formation of vibrations. Many different experiments have been made, especially in the field of technology, where the occurrence of self-excited vibrations was assessed depending on various technological parameters. However, if dynamic behavior of the mechanical structure is not taken into account at the same time, it is impossible to express the influence of these individual parameters in the mathematical formulation of the stability limit calculation. The current technological findings depend to a certain extent on the vibrating system of the machine on which the experiments were performed.

Based on a measured helix, our research is trying to determine dependence of the dynamic cutting force on both the cutting speed $v_{\mathrm{c}}$ and vibration frequency $\omega$.

The measured data showed a similar trend over the entire range of testing conditions. The center lines of the individual threads of the helix were not aligned. Endpoint discontinuity

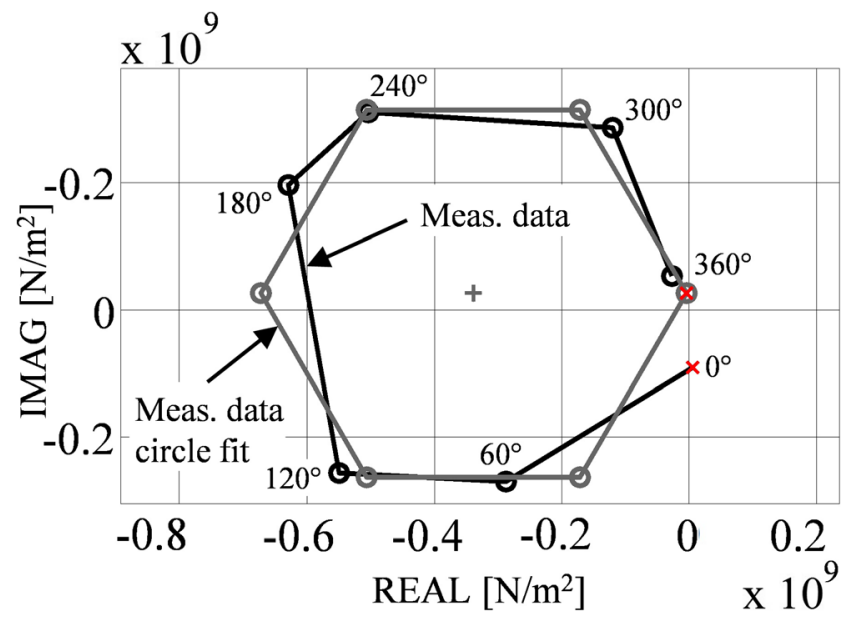

Fig. 16 Dynamic force $F_{y}$ in complex plane, $v_{\mathrm{c}}=30 \mathrm{~m} / \mathrm{min}, N=5$ 


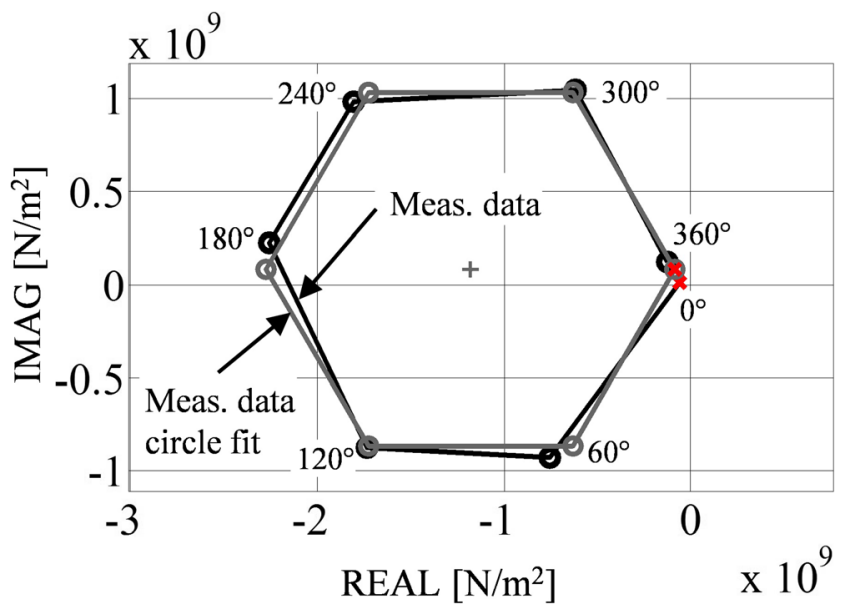

Fig. 17 Dynamic force $F_{\mathrm{z}}$ in complex plane, $v_{\mathrm{c}}=30 \mathrm{~m} / \mathrm{min}, N=5$

for $\psi=0$ and $\psi=2 \pi$ was identified in all measured threads in the direction of both the real and the imaginary axes. An example of measured data is provided in Figs. 16 and 17 and the frequency dependence pattern in Figs. 18, 19, 20, and 21 . Figure 18 clearly shows that the real component of the dynamic cutting force does not consist of the periodic component only and that its imaginary component, see Fig. 19, is not linearly dependent on frequency, as described by (9).

For illustration, Figs. 16 and 17 represent circular interpolation of the measured points using the least squares method. This interpolation is not entirely accurate as the measured projection of the thread shows a slight rotation relative to this interpolation.

The measured force signals in the $Y$ and $Z$ directions include components of all forces acting in the cut. From the measured frequency dependencies in the $Y$ direction, it can be concluded that there is at least one other force acting in the cut that has been unknown to us so far. In contrast, the force components in the $Z$ direction are only very little affected by frequency change.

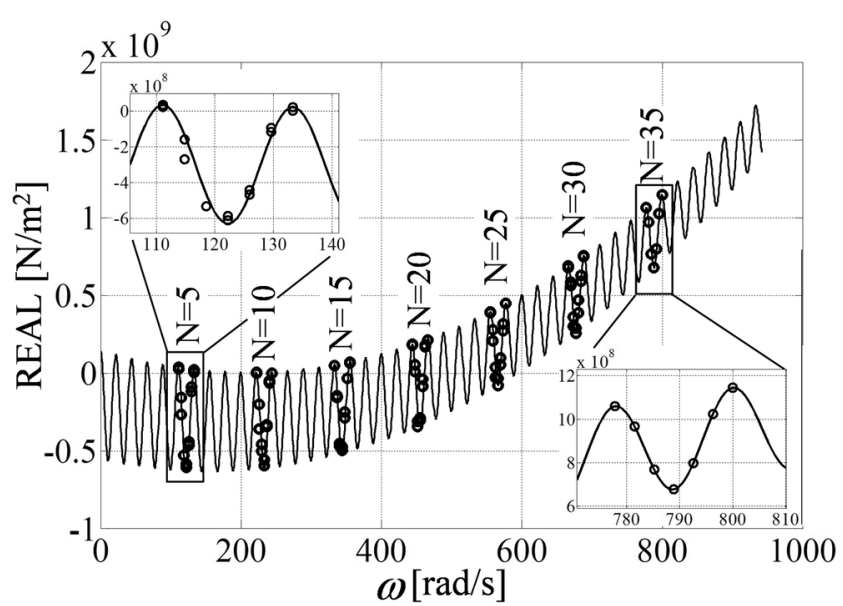

Fig. 18 Real component of total dynamic force $F_{\mathrm{y}}$ for $v_{\mathrm{c}}=30 \mathrm{~m} / \mathrm{min}$

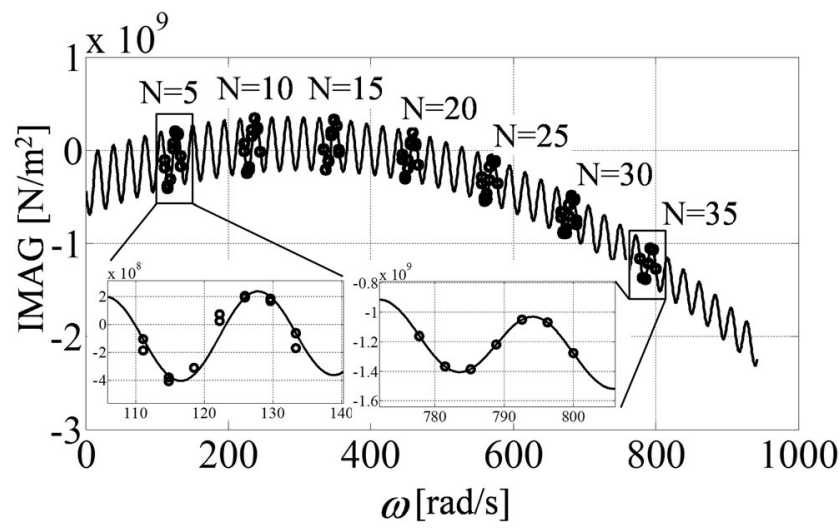

Fig. 19 Imaginary component of total dynamic force $F_{\mathrm{y}}$ for $v_{\mathrm{c}}=30 \mathrm{~m} /$ $\min$

Judging by the decrease in the amplitude of the periodic waveform in the $Y$ direction, a newly discovered wave cutting force arises which has a direction of the normal to the machined surface and counteracts force $\hat{F}_{o}$. Given the manner in which this force manifests itself, there is an idea that this force depends on the slope of the wave being cut. This would mean that the waves on the workpiece surface do not copy the vibrating motion but have a different shape.

However, it is not possible to determine this force or forces directly from the signal. In order to determine these forces, measurements must be taken under certain technological conditions that will influence the effect of the force. E.g., the effect of process damping forces can be influenced by the clearance or slope of wave on workpiece surface and the effect of tool wear forces can be increased or eliminated by using a tool with defined wear.

For calculation purposes, the measured data can be approximated by a suitable empirical model. There are two functions visible in the measured projections. A periodic function and a function having a rising and decreasing trend. For the purpose of calculating stability limits, we need to replace local measured points with a continuous waveform. It is necessary to

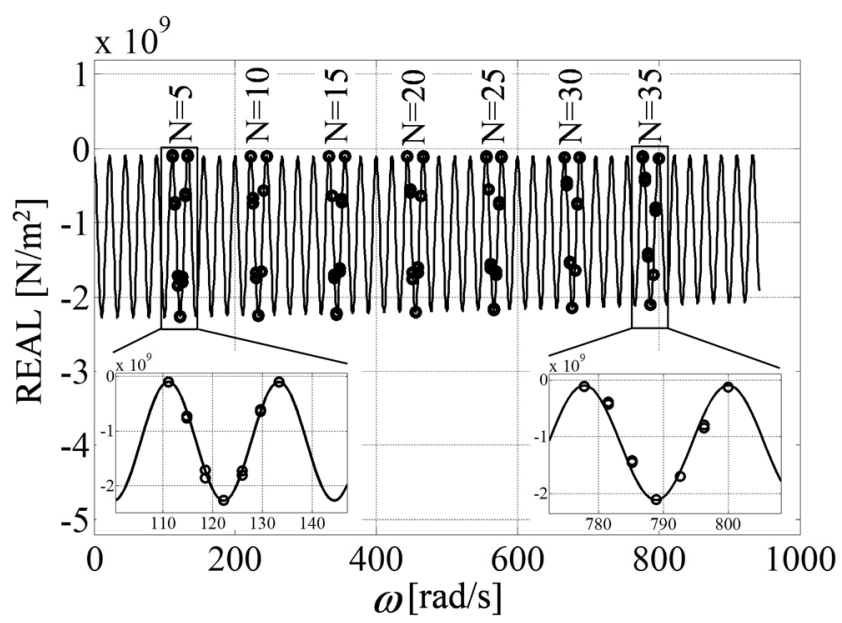

Fig. 20 Real component of total dynamic force $F_{\mathrm{z}} v_{\mathrm{c}}=30 \mathrm{~m} / \mathrm{min}$ 


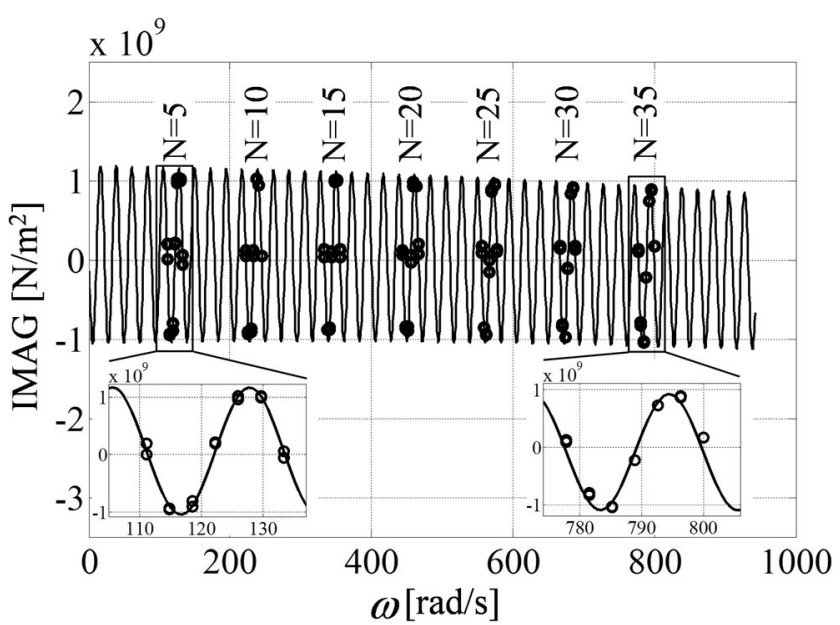

Fig. 21 Imaginary component of total dynamic force $F_{\mathrm{z}} v_{\mathrm{c}}=30 \mathrm{~m} / \mathrm{min}$

introduce an expression that accurately describes the measured behavior. Several experiments showed that the proposed empirical Eqs. (10) and (11) suited best. They very accurately capture the measurements depending on frequency and, in addition, they show that there is also another force in the cut that has a certain direction and behaves in a certain way, see Figs. 18 and 19. A helix was chosen for approximation of the measured data. The basis of the new model is Eq. (9) modified to respect amplitude damping of periodic behavior and nonlinear increase and decrease in the dynamic cutting force. A second-order approximation was chosen to describe the center line of the helix. The model also includes a measured static offset. Eq. (10) is used to describe the real dynamic force components, while Eq. (11) is used to describe the imaginary components.

$\operatorname{re}\left(\hat{K}_{y, z}\right)=D_{0}+$

$\left[D_{1}-\frac{60}{2 \pi \cdot v_{\mathrm{c}}} \cdot D_{2} \cdot \omega\right] \cdot[\cos (-\psi)-1]+$

$\frac{60}{2 \pi \cdot v_{\mathrm{c}}} \cdot D_{3} \cdot \omega+\left(\frac{60}{2 \pi \cdot v_{\mathrm{c}}}\right)^{2} \cdot D_{4} \cdot \omega^{2}$

$\operatorname{im}\left(\hat{K}_{y, z}\right)=D_{5}+$

$\left[D_{6}-\frac{60}{2 \pi \cdot v_{\mathrm{c}}} \cdot D_{7} \cdot \omega\right] \cdot \sin (-\psi)+$

$\frac{60}{2 \pi \cdot v_{\mathrm{c}}} \cdot D_{8} \cdot \omega+\left(\frac{60}{2 \pi \cdot v_{\mathrm{c}}}\right)^{2} \cdot D_{9} \cdot \omega^{2}$

where

$D_{0}, D_{1}, D_{2}, D_{3}, D_{4}, D_{5}, D_{6}, D_{6}, D_{8}$, and $D_{9}$ are the measured dynamic cutting resistance coefficients.

Table 1 shows the resulting approximation values of the proposed model for the tested frequency and cutting speed ranges.

Equations (10) and (11) replace the cutting process with two acting dynamic forces in the $Y$ and $Z$ directions. So, we know the direction of these forces and we can determine the stability limit for them.

However, if for calculation purposes we replace all the forces acting in the cut with just two resultant forces, we will lose the idea of the role that each of the individual forces plays in the cutting process. From a technological point of view, it is more advantageous to include the individual forces acting in the cut in the stability limit calculation. Each of the forces is dependent to a certain extent and has its own direction. Thus, each force will include a certain oriented compliance $\hat{\varphi}$ and the currently applicable methodology can be used to determine the stability limit.

Apart from the limit chip width $b$, the calculation also shows the vibrating frequency of the cutting process and the phase shift manifested by waves against the vibrations.

The experiments performed provide very good results for further processing. The task of further research will be to find ways of how to improve the new method to determine the behavior of individual dynamic forces, i.e., to determine individual dynamic coefficients, directions of individual dynamic cutting forces, and their dependence on technological conditions, cutting speed, and frequency.

The method can also be used to identify the size and direction of process damping. The following Figs. 22 and 23 show the waveforms of the real and imaginary components of the measured process damping in the $Y$ and $Z$ directions identified from the helix. The measured waveforms confirm the expected behavior of this force. At constant cutting speed, the force increases with the rising frequency and is in phase opposition to vibration. However, at $40 \mathrm{~m} / \mathrm{min}$, the real force component in the $Y$ direction changed its character significantly. The causes are unknown for the time being. Their clarification will be one of the goals of further research.

The empirical model described by Eqs. (10) and (11) is directly applicable to stability limit calculation. The coefficients $\hat{K}_{y}$ and $\hat{K}_{z}$ depend on the cutting speed and vibration frequency. When calculating the stability limit, it is necessary to consider the appropriate coefficient value for each cutting speed and frequency. If the dynamic coefficients include directional orientation, the stability limit can be determined similarly as in (5) from

$1=b \cdot\left(\hat{\varphi}_{y}(\omega) \cdot \hat{K}_{y}(\omega)+\hat{\varphi}_{z}(\omega) \cdot \hat{K}_{z}(\omega)\right)$

where

$$
\begin{aligned}
& \hat{\varphi}_{y}(\omega)=G_{y}(\omega)+j H_{y}(\omega) \quad \text { is the transfer function in the } \\
& \text { normal direction relative to the } \\
& \text { machined surface }(\mathrm{m} / \mathrm{N}) \text {; } \\
& \hat{\varphi}_{z}(\omega)=G_{z}(\omega)+j H_{z}(\omega) \quad \text { is the transfer function in the } \\
& \text { tangential direction relative to } \\
& \text { the machined surface }(\mathrm{m} / \mathrm{N}) \text {; }
\end{aligned}
$$


Table 1 Model parameters

\begin{tabular}{|c|c|c|c|c|c|c|}
\hline \multirow{2}{*}{$\begin{array}{l}V_{\mathrm{c}} \\
{[\mathrm{m} / \mathrm{min}]}\end{array}$} & \multirow[t]{2}{*}{ Direction } & \multicolumn{5}{|c|}{ REAL } \\
\hline & & $D_{0}\left(\mathrm{~N} / \mathrm{m}^{2}\right)$ & $D_{1}\left(\mathrm{~N} / \mathrm{m}^{2}\right)$ & $D_{2}(\mathrm{~N} / \mathrm{m})$ & $D_{3}(\mathrm{~N} / \mathrm{m})$ & $D_{4}(\mathrm{~N})$ \\
\hline \multirow[t]{2}{*}{20} & $Y$ & $\begin{array}{c}3.855 \mathrm{E}+ \\
08\end{array}$ & $1.085 \mathrm{E}+06$ & $\begin{array}{c}-4.558 \mathrm{E}+ \\
05\end{array}$ & $6.815 \mathrm{E}+03$ & $1.263 \mathrm{E}+07$ \\
\hline & $Z$ & $\begin{array}{c}1.251 \mathrm{E}+ \\
09\end{array}$ & $\begin{array}{c}-9.418 \mathrm{E}+ \\
04\end{array}$ & $7.081 \mathrm{E}+04$ & $\begin{array}{l}-5.104 \mathrm{E}+ \\
02\end{array}$ & $\begin{array}{c}-7.798 \mathrm{E}+ \\
07\end{array}$ \\
\hline \multirow[t]{2}{*}{30} & $Y$ & $\begin{array}{c}3.495 \mathrm{E}+ \\
08\end{array}$ & $5.452 \mathrm{E}+05$ & $\begin{array}{c}-4.305 \mathrm{E}+ \\
06\end{array}$ & $3.231 \mathrm{E}+04$ & $1.464 \mathrm{E}+08$ \\
\hline & $Z$ & $\begin{array}{c}1.102 \mathrm{E}+ \\
09\end{array}$ & $3.599 \mathrm{E}+05$ & $\begin{array}{l}-2.807 \mathrm{E}+ \\
05\end{array}$ & $9.886 \mathrm{E}+02$ & $\begin{array}{c}-8.625 \mathrm{E}+ \\
07\end{array}$ \\
\hline \multirow[t]{2}{*}{40} & $Y$ & $\begin{array}{c}5.102 \mathrm{E}+ \\
08\end{array}$ & $1.175 \mathrm{E}+06$ & $5.357 \mathrm{E}+07$ & $\begin{array}{c}-2.462 \mathrm{E}+ \\
05\end{array}$ & $\begin{array}{c}-2.099 \mathrm{E}+ \\
09\end{array}$ \\
\hline & $Z$ & $\begin{array}{c}1.307 \mathrm{E}+ \\
09\end{array}$ & $7.473 \mathrm{E}+05$ & $4.683 \mathrm{E}+06$ & $\begin{array}{c}-1.959 \mathrm{E}+ \\
04\end{array}$ & $\begin{array}{c}-3.181 \mathrm{E}+ \\
08\end{array}$ \\
\hline \multirow{2}{*}{$\begin{array}{l}V_{\mathrm{c}} \\
(\mathrm{m} / \mathrm{min})\end{array}$} & Direction & & & IMAG & & \\
\hline & & $D_{5}\left(\mathrm{~N} / \mathrm{m}^{2}\right)$ & $D_{6}\left(\mathrm{~N} / \mathrm{m}^{2}\right)$ & $D_{7}(\mathrm{~N} / \mathrm{m})$ & $D_{8}(\mathrm{~N} / \mathrm{m})$ & $D_{9}(\mathrm{~N})$ \\
\hline \multirow[t]{2}{*}{20} & $Y$ & $\begin{array}{c}3.933 \mathrm{E}+ \\
08\end{array}$ & $1.151 \mathrm{E}+06$ & $2.431 \mathrm{E}+06$ & $\begin{array}{c}-1.519 \mathrm{E}+ \\
04\end{array}$ & $\begin{array}{c}-7.046 \mathrm{E}+ \\
07\end{array}$ \\
\hline & $Z$ & $\begin{array}{c}1.363 \mathrm{E}+ \\
09\end{array}$ & $5.890 \mathrm{E}+05$ & $1.266 \mathrm{E}+06$ & $\begin{array}{c}-4.528 \mathrm{E}+ \\
03\end{array}$ & $\begin{array}{c}-6.941 \mathrm{E}+ \\
06\end{array}$ \\
\hline \multirow[t]{2}{*}{30} & $Y$ & $\begin{array}{c}3.295 \mathrm{E}+ \\
08\end{array}$ & $4.543 \mathrm{E}+05$ & $9.654 \mathrm{E}+06$ & $\begin{array}{c}-5.172 \mathrm{E}+ \\
04\end{array}$ & $\begin{array}{c}-3.800 \mathrm{E}+ \\
08\end{array}$ \\
\hline & $Z$ & $\begin{array}{c}1.120 \mathrm{E}+ \\
09\end{array}$ & $4.430 \mathrm{E}+05$ & $8.052 \mathrm{E}+04$ & $\begin{array}{c}-2.590 \mathrm{E}+ \\
03\end{array}$ & $6.093 \mathrm{E}+07$ \\
\hline \multirow[t]{2}{*}{40} & $Y$ & $\begin{array}{c}4.177 \mathrm{E}+ \\
08\end{array}$ & $4.065 \mathrm{E}+05$ & $1.673 \mathrm{E}+07$ & $\begin{array}{l}-1.083 \mathrm{E}+ \\
05\end{array}$ & $\begin{array}{c}-5.200 \mathrm{E}+ \\
08\end{array}$ \\
\hline & $Z$ & $\begin{array}{c}1.261 \mathrm{E}+ \\
09\end{array}$ & $5.396 \mathrm{E}+05$ & $\begin{array}{c}-3.314 \mathrm{E}+ \\
05\end{array}$ & $\begin{array}{c}-3.229 \mathrm{E}+ \\
03\end{array}$ & $8.084 \mathrm{E}+07$ \\
\hline
\end{tabular}

$\hat{K}_{y}(\omega)=A_{y}(\omega)+j B_{y}(\omega) \quad$ is the dynamic coefficient of the cutting forces in the normal direction (now complex)

$\left(\mathrm{N} / \mathrm{m}^{2}\right)$;

$\hat{K}_{z}(\omega)=A_{z}(\omega)+j B_{z}(\omega) \quad$ is the dynamic coefficient of the cutting forces in the

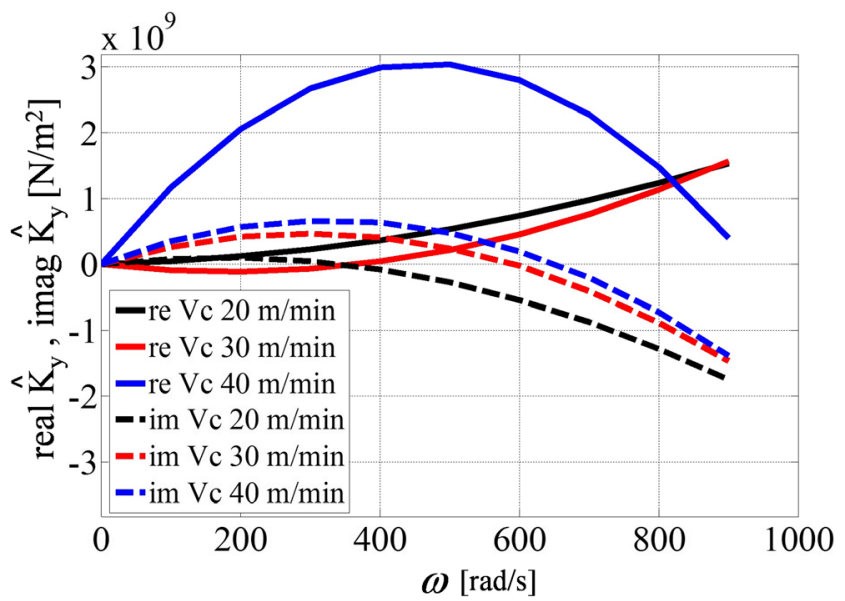

Fig. 22 Real and imaginary component in $Y$ direction tangential direction (now complex) $\left(\mathrm{N} / \mathrm{m}^{2}\right)$.

The limit chip width of cut $b$ is already complex, i.e., it has a real component (13) and an imaginary component (14). To determine the stability limit, it is necessary to find such frequencies $\omega$ where the imaginary

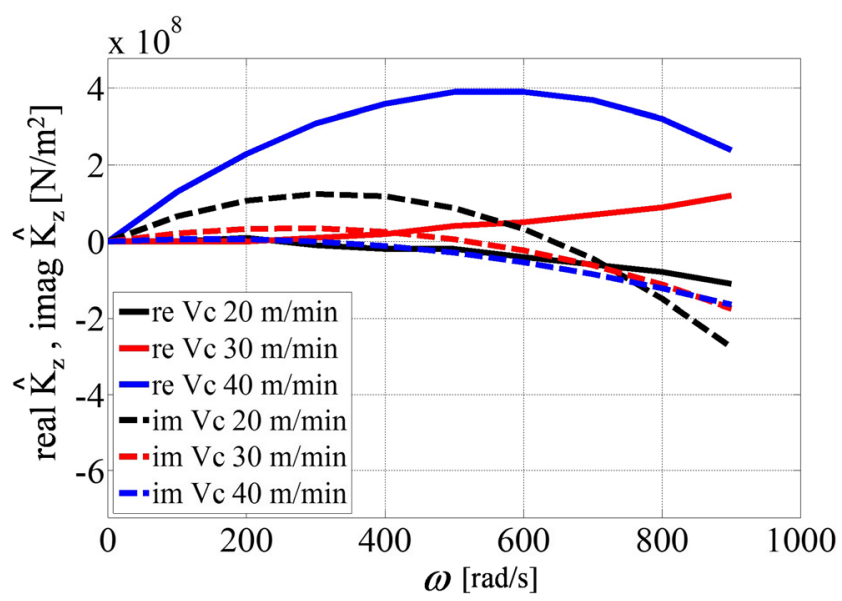

Fig. 23 Real and imaginary component in $Z$ direction 
component $b$ is 0 and it is necessary to select only those frequencies for which $b$ is a positive non-zero number. For a specific cutting speed, we get a set of frequencies $\omega$ and their respective limit widths $b$. As the limit width $b_{\lim }$ for a specific cutting speed, we choose the lowest of the values.

$$
\begin{aligned}
& A_{y} \cdot G_{y}-B_{y} \cdot H_{y}+ \\
& \operatorname{re}(b)=\frac{A_{z} \cdot G_{z}-B_{z} \cdot H_{z}}{\left[\begin{array}{l}
A_{y} \cdot G_{y}-B_{y} \cdot H_{y}+{ }^{2} \\
A_{z} \cdot G_{z}-B_{z} \cdot H_{z}
\end{array}\right]^{2}} \\
& {\left[\begin{array}{l}
A_{y} \cdot H_{y}-B_{y} \cdot G_{y}+ \\
A_{z} \cdot H_{z}-B_{z} \cdot G_{z}
\end{array}\right]^{2}} \\
& A_{y} \cdot H_{y}-B_{y} \cdot G_{y}+ \\
& \operatorname{im}(b)=-\frac{A_{z} \cdot H_{z}-B_{z} \cdot G_{z}}{\left[\begin{array}{l}
A_{y} \cdot G_{y}-B_{y} \cdot H_{y}+ \\
A_{z} \cdot G_{z}-B_{z} \cdot H_{z}
\end{array}\right]^{2}+} \\
& {\left[\begin{array}{l}
A_{y} \cdot H_{y}-B_{y} \cdot G_{y}+ \\
A_{z} \cdot H_{z}-B_{z} \cdot G_{z}
\end{array}\right]^{2}}
\end{aligned}
$$

It should be noted that Eqs. (10) and (11) are also a $\psi$ function. However, the $\psi$, function is also a $\omega$ frequency function, see Eq. (3). Within the considered frequency range, the $\psi$ function takes values in the $\langle 0,2 \pi\rangle$ range only. This property is caused by the behavior of the $N$ parameter. Since we have obtained only a limited number of values for the time being, it makes no sense to present the calculated stability limit as it would only cover a very narrow range of cutting speeds.

The results of the performed experiments clearly show that it is impossible to identify the behavior of dynamic forces from static tests and to use the static coefficient $K_{\mathrm{C}}$ to calculate the stability limit. With regard to the measured frequency dependence of the cutting forces, it can also be confirmed that the forces cannot be even identified at the stability limit where the system vibrates at around a single frequency given by the characteristics of the vibrating system.

To clearly identify the new force, additional measurements will have to be made under different technological conditions. The measured results clearly show the importance of application of complex coefficients capable of a very simple description of the mutual phase relations between tool vibration and dynamic cutting forces. When summarizing the results obtained so far, we must admit that such a type of research brings along many new challenges. On the other hand, as we still have a very limited idea of what is actually happening in the cut, this approach opens up new possibilities for us to explore the dynamic cutting process.

\section{Conclusion and future work}

The experiment proved that the dynamic cutting force depends not only on cutting speed but also on vibration frequency.

The experiment suggests that in addition to the forces $\hat{F}_{i}$ and $\hat{F}_{o}$, there are process damping forces and a force (or forces) dependent on wave cutting acting in the cut. The new methodology used to process the measured results showed that a helix, instead of the circle used so far, can be used to describe the force-frequency behavior.

By comparing the results at speeds of $20-40 \mathrm{~m} / \mathrm{min}$, it can be said that the force in the tangential direction ( $Z$ direction) is roughly the same for all speeds and very little dependent on vibration frequency and cutting speed. Cutting speed and frequency mostly affect the force in the normal direction $(Y$ direction).

From the identified center lines of the measured helices, it is possible to directly obtain the behavior of the force called process damping. By comparing the waveforms of the real and imaginary components at speeds of $20-40 \mathrm{~m} / \mathrm{min}$, it can be assumed that the mechanics of cutting and thus the resulting cutting forces change considerably at $40 \mathrm{~m} / \mathrm{min}$. The largest deviations are in the real process damping component for the speed of $40 \mathrm{~m} / \mathrm{min}$.

This force has a $Y$-normal direction and is dependent on frequency and cutting speed. This force arises in cutting waves and counteracts the already known force $\hat{F}_{o}$. The force direction can be deduced from the decrease in the amplitude of periodic behavior with frequency. This force may be related to the slope of the cut waves or the formation of chips. Description of chip formation mechanics during vibration is a task requiring expertise especially in the field of technology.

This article introduces an improved method of controlled cutting tool vibration, which, unlike the original method, also considers the effect of frequency on the dynamic cutting process. The controlled cutting tool vibration method is the most appropriate way of how to reliably identify and track dynamic forces acting in a cut.

Future research will in the first phase include additional measurements to refine the conclusions obtained. It will be necessary to establish why the waveform for the speed of $40 \mathrm{~m} / \mathrm{min}$ is so much different. The research will then focus on identifying the conditions for the emergence and existence of the newly discovered force, including formulation of its mathematical description. The aim of further research should be to investigate dynamic cutting forces for a greater range of technological conditions, materials, and cutting tools.

Acknowledgements The research was supported by the Technology Agency of the Czech Republic from the funds earmarked for the TE01020075 Competence Centre project. 


\section{References}

1. Altintas Y (2012) Manufacturing automation: metal cutting mechanics, machine tool vibrations, and CNC design, 2nd edition, Cambridge University press, 2012, New York. ISBN 978-1-10700148-0

2. Cheng K (2008) Machining dynamics: fundamentals, applications and practices. Springer, London ISBN 978-1-84628-367-3

3. Tlusty J, Polacek M (1963) The stability of the machine tool against self-excited vibration in machining. ASME Int Res Prod Eng:465474

4. Tlusty J (1978) Analysis of the state of research in cutting dynamics. Ann CIRP 27:583-589

5. Goel B (1976) Measurement of dynamic cutting force coefficients. Dissertation, McMaster University. https://macsphere.mcmaster.ca/ handle/11375/8095>. Accessed 18 June 2014

6. Rao SB (1977) Analysis of the dynamic cutting force coefficient. Dissertation McMaster University. https://macsphere.mcmaster.ca/ handle/11375/8826>. Accessed 18 June2014

7. Nigm MM, Sadek MM (1977) Experimental investigation of the characteristic of dynamic cutting process. ASME J Eng Ind 99: 410-418. https://doi.org/10.1115/1.3439252

8. Heczko O (1980) New method for testing the dynamic cutting force coefficients. Dissertation, McMaster University. https://macsphere. mcmaster.ca/handle/11375/17929. Accessed 19 August 2015

9. Ahn TY, Eman KF, Wu SM (1985) Identification of the transfer function of dynamic cutting processes - a comparative assessment. Elsevier Int J Mach Tool Des Res 25:75-90. https://doi.org/10. 1016/0020-7357(85)90059-9

10. Minis IE, Magrab EB, Pandelidis IO (1990) Improved methods for the prediction of chatter in turning, part 2: determination of cutting process parameters. ASME J Eng Ind 112:21-27. https://doi.org/10. $1115 / 1.2899291$

11. Insperger T, Stepan G (2011) Semi-discretization for time-delay systems: stability and engineering applications. Springer, New York ISBN 978-1-4614-0334-0
12. Wu DW, Liu CR (1985) An analytical model for cutting dynamics. Part 1: model building. ASME J Eng Ind 107:107-111. https://doi. org/10.1115/1.3185972

13. Altintas $\mathrm{Y}$, Eynian M, Onozuka H (2008) Identification of dynamic cutting force coefficients and chatter stability with process damping. CIRP Ann Manuf Technol 57:371-374. https://doi.org/ 10.1016/j.cirp.2008.03.048

14. Budak E, Tunc LT (2010) Identification and modeling of process damping in turning and milling using a new approach. CIRP Ann 59:403-408. https://doi.org/10.1016/j.cirp.2010.03.078

15. Xiao W et al (2014) A method of using turning process excitation to determine dynamic cutting coefficients. Elsevier Int J Mach Tools Manuf 87:49-60. https://doi.org/10.1016/j.ijmachtools.2014.08. 002

16. Sniegulska-Gradzka D, Nejman M, Jemielniak K (2017) Cutting force coefficients determination using vibratory cutting. Procedia CIRP 62:205-208. https://doi.org/10.1016/j.procir.2016.06.091

17. Bach P, Polacek M, Chvojka P, Drobilek J, Svoboda O (2013) A comparative analysis of lower speed chatter behaviour. MM Sci J 2013:434-440. http://www.mmscience.eu/content/www mmscience cz 201314(1).pdf. Accessed 7 Jan 2014

18. Nigm MM, Sadek MM, Tobias SA (1977) Dimensional analysis of the steady state orthogonal cutting process. Elsevier Int J Mach Tool Des Res 17:1-18. https://doi.org/10.1016/0020-7357(77)90023-3

19. Munoa J, al e (2016) Chatter suppression techniques in metal cutting. CIRP Ann 65:785-808. https://doi.org/10.1016/j.cirp.2016. 06.004

20. Peters J, Vanherck P, Van Brussel H (1971) The measurement of the dynamic cutting coefficient. Elsevier CIRP Ann Manuf Technol 21: $129-136$

Publisher's note Springer Nature remains neutral with regard to jurisdictional claims in published maps and institutional affiliations. 